\title{
The Effect of Hepatic-Artery Geometry on the Particle Release Map
}

\author{
Yuki Tsuzuki ${ }^{1}$, Emilie Roncali ${ }^{2}$,Simon R. Cherry ${ }^{2}$, Ralph C. Aldredge ${ }^{1}$ \\ ${ }^{1}$ Department of Mechanical \& Aerospace Engineering \\ University of California, Davis \\ ${ }^{2}$ Department of Biomedical Engineering \\ University of California, Davis \\ One Shield, Davis, USA \\ ytsuzuki@ucdavis.edu
}

\section{Extended Abstract \\ Objective}

The purpose of this research is to computationally analyze the patient-specific scenario for radioembolization (RE) by focusing on the changes in the particle-release map (PRM). RE is an efficient internal radiation therapy to treat liver cancer by delivering radioactive yttrium 90 microspheres in the hepatic artery and directly targeting the tumor(s) with radiation. Current tools to determine the yttrium-90 activity to inject are not adapted to the heterogeneous distribution of the microspheres and provide limited information to the clinicians to make a decision. Currently, computational fluid dynamics (CFD) in the hepatic arterial tree is limited to a generic model as seen in Kennedy et al. [1] and has not been applied to study differences between patients such as hepatic-artery diameter-size variations observed in Ishigami et al. [2], leading to an inefficient microsphere delivery due to patient-specific changes in the PRM. To increase the accuracy of the PRM, we propose to computationally analyze the PRM of the branched systems, considering changes in the vessel diameter, angle between branches, and branched positions in the region of the hepatic artery, to accurately estimate the RE delivery for patient specific geometries.

\section{Scope}

The scope of the research is to use CFD to analyze the PRM by using rat liver ex vivo data of the hepatic artery. The geometry is modified for possible case scenarios accounting for the angle between branches, scale of the vessel diameter, and branched positions for multi-branched scenarios.

\section{Results}

The results are based on computational analysis using ex vivo data of casted rat livers, imaged with microCT at 30micron spatial resolution. The hepatic arterial tree was segmented from the microCT images, and was scaled up to match the human hepatic-artery size (inlet diameter of $\sim 6 \mathrm{~mm}$ ) as specified in Ishigami et al. [2]. Two meshes are constructed. Case 1 consists of only six outlets and one inlet and is maintained as a proof-of-concept simulation, while Case 2 represents the first bifurcation of Case 1, to test influences of vessel diameter and angle on the PRM. The microsphere size is $30 \mu \mathrm{m}$, similar to that of TheraSphere glass microspheres (BTG Interventional Medicine). A total of 10,000 microspheres are injected uniformly at the inlet. The CFD simulation is conducted using the open-source software OpenFOAM, using the Lagrangian particle method. Each microsphere is tracked from its inlet to its respective outlet to provide the PRM for each scenario. The results from Case 1 show the PRM to be in discrete sections, similarly observed in Kennedy et al [1]; however, the differences in the branched positions, diameter size, and angle from the generic case show deviation from the PRM of Kennedy et al. [1]. Results from Case 2 show that the effect of a 60-degree change in angle results in a difference within $\sim 1 \%$ while a scale reduction of $60 \%$ of the connected branch shifts the PRM by a similar $60 \%$ area reduction. Further analysis will be conducted on the effects of differences in the vessel diameter, angle and branched positions for multi-branched scenarios in order to estimate the effect on the PRM. 


\section{References}

[1] A. Kennedy, C. Kleinstreuer, C. A. Basciano and A. Dexarn, "Computer modelling of 90Y microsphere transport in the hepatic arterial tree to improve clinical outcomes," Int. J. Radiat. Oncol., vol. 76, no. 2, pp. 631-637, 2010.

[2] K. Ishigami, Y. Zhang, S. Rayhill, D. Katz, and A. Stolpen, "Does variant hepatic artery anatomy in a liver transplant recipient increase the risk of hepatic artery complications after transplantation," Am. J. Roentgenol. vol. 185, pp. 1577-1584, 2004. 